\title{
Study on the Training Strategy of Students' Practical Ability in Education Technology Major
}

\author{
Yan YuE*, Wang Haiyan \\ Institute of Education, Shaanxi Normal University, Xi'an, China \\ Email address: \\ 438759641@qq.com (Yan YuE) \\ ${ }^{*}$ Corresponding author
}

To cite this article:

Yan YuE, Wang Haiyan. Study on the Training Strategy of Students' Practical Ability in Education Technology Major. Science Discovery. Vol. 6, No. 5, 2018, pp. 380-385. doi: 10.11648/j.sd.20180605.21

Received: September 29, 2018; Accepted: October 15, 2018; Published: October 18, 2018

\begin{abstract}
With the development of information technology education and teaching reform, professional and educational technology have also been developed, educational technology is based on practical application of discipline on the basis of social practice ability of educational technology undergraduate raised higher and higher requirements. However, many colleges and universities exist in the practical ability of lack of cause of educational technology specialty current widespread concern is to develop educational technology professional students' practical ability, Students and teachers are in a confused period, we do not know how to carry out effective teaching and learn. So I think many students graduate without a good job, the national employment requirements and student capacity want to violate through the analysis of the connotation of practical ability, so in my opinion content herein educational technology practical ability and current educational technology major in the culture is present at the practical ability aspects and educational technology practice capability requirements were discussed, and finally by improving the training plan, a reasonable set of theoretical knowledge, strengthen experiment teaching, improve the practical ability to strengthen students of the corresponding training program innovation and practical ability to improve the educational technology majors in practical measures ultimately promote overall development of students.
\end{abstract}

Keywords: Educational Technology Major, Practical Ability, Training Ways

\section{教育技术学专业学生实践能力培养策略研究}

\section{间宇娥，王海燕}

陕西师范大学, 教育学院, 西安, 中国

\section{邮箱}

438759641@qq.com(间宇娥)

\footnotetext{
摘要: 随着教育信息化与教学改革的深入, 教育技术学专业也得到发展, 教育技术学是建立在实践应用基础上的学科, 社会对教育技术学本科生的实践能力提出越来越高的要求, 然而很多高校在实践能力培养方面存在着欠缺, 很多学生 毕业不能很好的就业, 国家就业人才要求与学生能力想违背, 导致教育技术学专业目前普遍关注的问题是教育技术学 专业学生实践能力的培养，本文通过分析了实践能力的内涵、教育技术学实践能力的内涵以及当前教育技术学专业在 培养学生实践能力方面存在的问题, 然后对教育技术学实践能力需求进行了探讨, 最终提出了完善培养计划、合理设 置理论知识体系、加强实验课教学、提高实践能力、加强学生的创新能力和实践能力等相应的培养方案来提高教育技 术学专业学生实践能力措施, 最终促进学生全面发展。
} 
关键词: 教育技术学专业, 实践能力, 培养途径

\section{1. 引言}

随着教育信息化的快速发展, 教育技术学专业也得到 了发展, 根据不太准确的统计, 目前中国拥有教育技术学 本科专业的高校达到 200 多个, 而在 90 年代还达不到 40 个, 这足以可以看出教育技术学专业本科生就业形势越来越 严峻, 目前来说, 就业单位关注的是毕业生的实践能力, 要求越来越缩短岗前培训, 减少用人成本, 因此实践能力 对本科生就业而言是必不可少的, 但是如何培养学生的实 践能力是当今教育界的热点话题, 也是教育技术学的改革 方向。很多学者对学科建设、培养目标、课程体系等进行 了不断的反思和不解的努力, 在学生学习方面重中之重的 实践能力的培养上, 他们同样也进行了探索与研究, 例如: 徐小双老师根据地方院校的特点对实践教学进行了探讨, 而衷克定老师以北京师范大学的实践为背景基础构建了 一套实践能力培养方案。[1]这些研究对教育技术专业学生 实践能力的培养提供了指导和帮助, 然而很少甚至没有提 出完整的实践能力培养的教学、实践活动的具体组织策略。 因此, 本文在探究实践能力的内涵、特征的基础上,结合 我院的教学实践, 建构内容关联且分层的理论与实践课程 体系, 进一步完善校内外实训基地,促进学生发展的评价 机制等具体的实践能力培养策略。

\section{2. 教育技术学实践能力}

\section{1. 实践能力和教育技术学专业实践能力的内涵}

\subsection{1. 实践能力的内涵}

实践跟实践能力密不可分的, 只要发展实践就离不开 实践能力的发展, 这样可以认为人的实践发展过程就是实 践能力发展的过程, 即: 人的客观活动都可以认为是实践 的活动。在心理学上, 不同的学者对实践能力提出不同的 看法, 但他们集中研究的实践智力, 也就是行为上的能力 展现。仅仅从字面上解释, 实践能力就是实践的能力, 也 就是解决实际问题的能力。在我国实践能力分为广义跟狭 义之分, 广义是指人在解决生活或者工作的实际问题而表 现的综合能力。狭义是动手操作能力、实验的能力、技能 的动手能力等肢体性活动能力。对于利用不同的方法来划 分实践能力, 实践能力的分类不同, 通过实践能力解决问 题的层次划分，分为基础实践能力和专业实践能力。基础 实践能力比较广包括沟通交往能力、语言表达能力、团队 合作能力等是解决综合问题的基础; 专业实践能力是指从 事某项专门或者特殊的活动必不可少的能力, 如计算机、 英语、医学等技能型专业要求的以特殊学科知识为背景的 实践能力。[2]

\subsection{2. 教育技术学专业实践能力的内涵}

如今, 多媒体计算机的快速普及, 改变了传统的教 学环境, 丰富了教学资源, 促进了教育技术的发展, 在
实践中形成新的学习资源和环境。随着计算机成为各行 各业必不可少的设备, 社会越来越需要在教育理论指导 下进行教与学的设计、开发、管理和应用, 把教育理论 与教育技能结合起来, 来培养教育技术学专业人才 [3]教 育技术学专业学生必须具备教学设计和信息处理能力并 且具备对学习过程和学习资源的设计、开发和研究的能 力。因此, 教育技术学专业的本科生应该掌握丰富和全 面的实践能力, 具体是: (1) 教学设计能力。对教育技 术学专业来说, 教育技术学专业学生培养方向其中之一 是信息技术教师, 因此, 教育技术学专业的学生必须要 具备一定的设计能力。具体包括对教学设计理论技能的 掌握、教学设计实践能力的掌握, 而实践能力更为重要。 教育技术学专业学生必须明白设计具体的步骤并且要熟 练使用和选择多媒体资源, 对于不同的课程要学会灵活 应用, 要求掌握教学系统、教学过程、教学产品等设计 的基本理论和方法的能力, 最终达到教学最优化。（2) 动手实践能力。教育技术学专业开设的实践类课程较多, 比如: 摄影技术、影视编辑技术, 但是咋样把理论运用 在实际的操作上, 是学生必须掌握和培养的技能。(3)创 新能力。(4)教育技术学专业研究能力。教育技术是理论 和实践相结合的学科, 需要学生必须具备一定的分析问 题、解决问题的能力和科学研究的能力。

\section{2. 教育技术学专业在培养学生实践能力方面存在的问 题}

教育技术学是一门实践性比较强的学科、对于高校在 培养教育技术学专业实践能力存在的问题, 经过深入分析, 主要存在以下几方面的问题:

\subsection{1. 课程设置杂而不精}

中国教育技术学专业培养目标大多数是通过美国 AECT'94定义改编而成, 表述类似“培养从事教学资源和 教学系统的设计、开发、运用、管理和评价的教育技术学 科专门人才”, 缺乏本土特色, 并且各大高校的特色也不 突出。在课程内容上, 大多数高校是以教育部教育技术学 专业教学指导委员会规定的五大专业方向, 即: 教育技术 学、数字媒体技术、信息技术教育、现代远程教育、教育 软件、综合设置课程。看起来样样都有, 但是样样都不深 入, 教学内容宽而不深, 显然达不到培养专业人才的条件。 事实上各大不同水平、不同性质、不同地域的学校拿着一 样的培养方案进行教学, 根本没有根据培养目标在教学内 容方面有侧重点, 也没有明确的培养目标, 结果就是学生 毕业工作适应性差, 而且能力不够。

\subsection{2. 专业课程实践少, 学生缺乏实训和实习机会}

很多高校制定教育技术学专业时, 理论课时多而实 践课时少, 很多学校的实验课达不到总课程的 $30 \%$, 学 校和老师没有足够的重视实验课程, 导致学生在实践方 面缺乏, 实验效果不佳。学生实践在技术方面比高校更 
新技术和知识更快; [4]这样如果可以跟企业合作安排大 四学生去他们单位实习, 对双方都有好处, 可以达到双 赢。但是很多学校只安排在中小学实习, 几乎没有在IT 企业实习和训练。

\subsection{3. 教育技术学专业教师教育实践的欠缺}

很多高校的教育技术学专业师资来自于高校硕士或 博士, 他们理论知识非常扎实, 但是在专业方面的从业经 验较为缺乏, 所以就会导致学生实践时无的放矢。还有很 多地方性本科院校的教育技术学由于经费不足问题, 较少 到企业或者高校进行有针对的培训和进修, 这样就会导致 教师在专业技能和理论知识不能有效的训练和更新, 就抑 制教师专业发展，不能更好地提高教学质量。

\subsection{4. 实践环节薄弱}

学习教育技术最好的方式就是应用教育技术。但是在 很多教育技术实验或实践过程中，教育技术手段用的并不 充分, 甚至一些仍然用传统的实验方式进行, 并没有利用 类似虚拟仿真软件来解决设备不足、效果不佳的问题。有 些实验, 比如服务器配置、网络管理, 如果可以让学生事 先通过虚拟仿真软件课前学习一遍实践过程, 然后再进行 真实验操作, 这样学生就能够很好地抓住实验难点、重点, 及时处理发现的问题, 提升实验效果。

\section{3. 教育技术学专业学生实践能力需求}

从专业建设和教育改革的角度可以看出教育技术学 专业学生所需的实践能力是很丰富的, 笔者认为有以下几 种需求:

\section{1. 教学系统设计能力}

这里的教学系统是指不同层次的、比较大的、比较综 合和复杂的教与学的系统, 包括了促进学生学习的内容、 条件、资源等, 例如远程教育系统、学校教学系统、课程 的培养方案、职业教育等。这里的设计能力主要是指通过 把传播理论、学习理论和教学理论三者系统地应用于解决 教学实际问题中经过验证、能实现预期功能的教与学系统。 可以直接运用于教学过程, 完成一定的教学目标的教学资 源（如印刷教材、声像教材、学习指导手册）, 也可以是 对一门课的大纲与实施方案, 或者是对一个单元、一节课 教学计划的详细说明。如: 能制定出适宜本学校的具体的 课程培养目标和教学计划, 能建立远程教学质量检查和评 估制度, 能制定出人员的培训计划和制度等较高水平的设 计活动。

\section{2. 教育信息处理的能力}

知道教育信息产生、传播的规律，了解信息表征元素 (文本、图片、动画、音频、视频) 在教学中的特点和作 用, 掌握教育信息获取、储存、加工、利用、评价和创新 的理论和方法。然后根据自己所学的理论知识能够辨别信 息真假, 进行处理信息。

\section{3. 教育技术学专业研究能力}

教育技术是理论和实践相结合的学科, 需要学生必须 具备一定的分析问题、解决问题的能力和科学研究的能力。 学生通过学习教育技术学研究方法这门课程后有一定的 理论知识, 加上学生自己的实践经验, 最后具有一定的研 究能力。

\section{4. 媒体 (资源) 开发、应用的能力}

了解教学媒体(资源)的分类、特性、在教学中的作用, 掌握教学媒体 (资源) 的选择、应用、管理和评价的方法, 必要时能够设计、开发适合教学使用的媒体和资源。比如 以多媒体与网络技术实践开发为例, 需要掌握以下几种理 论知识和实践经验, 才可以开发多媒体与网络技术。

（1）掌握教育技术学科的基本理论和知识, 了解教 育技术学科多媒体技术、网络技术在教育应用的发展动态, 发展前景。

（2）全面了解与所学专业相关的其他学科的基础知 识; 掌握基本的科学研究方法, 具有较宽的知识面和较高 的人文、科学素养。

（3）具有比较扎实的网络及多媒体专业知识; 具有 熟练的网络及多媒体技术应用技能; 掌握计算机网络组建、 组织实施安装和维护, 网络系统管理维护的知识与实现技 术; 具备较强的实际工作能力。

（4）熟悉计算机信息网络系统的结构组成及性能特 点, 掌握计算机网络最新的网络工程设计、工程施工技术 和施工管理技术。

（5）具有计算机网络安全管理、网站设计与维护、 中小型管理信息系统开发维护的能力; 多媒体项目服务与 设计开发、网络产品营销的能力。

\section{5. 创业就业能力}

通过以适应各类各级教育教学活动、企事业单位工作 场景及自主创业过程遇到的实际问题解决为核心，修改基 于良构验证性与设计性实验养成的问题解决图式, 激励和 巩固本科毕业生在实际工作中遇到实际问题能够独立处 理的信心与能力, 提升毕业生入职初期的职业适应与工作 能力。

除上述专业能力外, 教育技术学学生和其他专业的学 生一样, 他们还应该具备较高的政治素养、完善的人格以 及持续更新、补充和完善知识结构的意识和能力。

\section{4. 教育技术学专业学生实践能力培养措施}

\section{1. 加强实验课教学,提高实践能力}

教育技术学的实验内容包括信息技术与计算机专业, 教育学专业等课程的内容, 其内容具有广泛性, 具体分为 操作性实验、制作性实验、教学技能性实验。操作性实验 是让学生熟练掌握教学软件的操作和使用; 制作性实验是 让学生熟练掌握应用软件的编制方法和技巧; 教学技能训 练性实验是让学生利用多媒体组合教学设计的方法来解 决课程教学时遇到的实际问题。实践教学分为相对独立的 
基础实验和综合实验两部分, 基础实验中有部分操作需要 依附课程教学, 即需要根据课程进度安排对应的实验, 这 些实验有针对性, 目的性较强, 促进学生掌握系统的课堂 知识, 掌握多媒体使用; 制作性实验和教学技能训练性实 验注重整体化设计, 培养学生的综合能力, 分析问题、解 决问题能力和创新运用能力。为了提高实验教学, 从以 下几方面进行。

\subsection{1. 合理设置实验课, 提高学生实践能力}

提高学生的实践能力有效方法之一是合理安排实验 课, 这样可以增加实验机会, 学生根据自己的特点选择实 验课, 有利于个性的培养和形成良好的研究氛围, 促进学 生实践能力的培养。具体可以分为以下几个实验方法来安 排实验课, 提高学生的实践能力。

\section{(i). 协同性实验}

以合作学习理论为基础, 以3-4人组成项目专题, 在 教师的引导下, 每一组给出一个实验课题, 然后学生进行 集体探讨, 提出实验步骤和方法, 共同完成实验任务, 教 师帮助学生解决疑难问题和引导学生思考。学生在实验中 不断地修改和完善实验内容、步骤、方法。最后学生一起 完成实验任务, 每个人根据自己的看法, 真实的完成实验 报告。

\section{(ii). 综合性实验}

在一门和几门实验课程临近结束时, 学生提出跨课程 的实验题目, 在校内外实训基地, 进行有针对性的训练和 实践, 也可以把班级分成若干小组, 每个小组有一至二名 教师进行指导。通过这些实践, 提高了学生分析解决实际 应用问题和创新应用的综合培养。

\section{(iii). 探究性实验}

根据布鲁纳的“发现学习”原理, 学生通过“发现法”来 掌握学科的基本机构, 建设探索实验环境, 学生主动参与 到实验教学过程中, 自己设计实验, 自行观察、分析和总 结实验现象, 发现实验结果, 培养探索精神, 从中获得成 功的喜悦感。

\subsection{2. 提高实验课教师的实践能}

教师在实践教学中起着引导者和组织者的角色, 所以 教师团队建设在实践教学必不可少的, 指导教师需要是 “双师型”人才, 不仅具有理论基础还必须具有较强的实践 能力。学校鼓舞教师到业务单位和进修单位学习, 来进一 步完善教师的知识结构, 提高实践教学能力。

\subsection{3. 建设实验教学效果的评价体系}

实验教学质量评价依教学目标分类, 有过程评价和结 果评价。

（1）根据本校实际情况, 然后通过调研, 最后确定 评价的各个要素。

（2）首先规定评定指标, 然后把指标定量化, 这样 可以客观地反映学生对知识和技能的掌握情况, 为了提高 学生实验教学质量综合评价的准确性, 排除一些人为因素。

\section{2. 完善培养计划, 合理设置理论知识体系}

理论知识是实践的基础, 没有理论知识指导的实践是 盲目的实践, 所以合理设置理论知识体系, 完善人才培养 计划。

\subsection{1. 根据学科的最新发展, 合理调整课程结构}

信息技术的发展与日俱进，技术半衰期将会越来越短 暂, 教育技术学专业的知识结构更新越来越迅速。如利用 昨天的技术培养今天的学生, 这将会导致教师下岗; 利用 明天的技术来培养今天的学生, 才会赢得未来。所以, 根 据高中信息技术课程的需求, 因为总课时是有限的, 所以 对课程结构进行调整, 删除一些杂乱的课程, 比如普通物 理、模拟电子线路课程, 而新加信息技术教学法、人工智 能和考研涉及的课程, 提高学生专业技能和职业意识, 重 新不断地改变专业课程的知识、能力和素质结构, 来优化 培养人才方案。[5]

\subsection{2. 通过以能力为本位来细化课程设置}

利用心理学来解释能力, 它与大脑的机能有联系, 是 通过人们展现出来有可能解决问题的个体心理特点, 能力 的必要条件是要求完成所布置的任务, 达到一定的目标。 能力可以通过运用智能、知识、技能进行反复训练得到的。 [6]由此可知, 教育技术学专业学生实践能力的培养是需要 以一定的知识系统和技能为前提的, 所以课程设置必须要 体现一定的系统性。利用影视方向的课程来将具体课程设 置细化。包括专业基础课、专业选修课和专业必修课, 专 业基础课有色彩与构图、摄影; 专业必修课有电影摄像、 非线性编辑; 专业选修课有电视教材编导与制作、电视节 目制作。首先是专业基础课的学习, 其次是专业必修课的 学习, 最后是专业选修课的学习, 这样设置可以促进学生 丰富和发展知识体系, 达到提高和运用实践能力。

\section{3. 整合教育技术资源，优化实践培养}

教育技术资源对学生实践培养具有重要的条件基础, 所以必须合理利用资源, 来提高学生的动手实践能力

\subsection{1. 整合教育技术物质资源, 优化实践教学环境}

教育技术学专业应该具备一定的实践能力, 学生实践 能力的培养需要良好的环境, 需要建立实验室比如计算机 网络、多媒体教学、电视摄像、非线性编辑、摄影等等, 虽然经费投入较大, 然而现代教育技术中心的日常工作也 需要这些设备。[7]如果将学校教育技术基础设施建设和教 育技术学专业实验室建设结合在一起, 把现代教育中心的 工作环境作为教育技术学专业学生的校内实践基地, 把很 多专业实验室的工作移植到现代教育技术中心的相关科 室。这样就可以为学校节省许多专业建设经费, 提高现代 教育技术中心设施设备的利用率, 同时也可以创造丰富、 宽敞的实践教学环境。[8]

\subsection{2. 整合教育技术人力资源, 推行本科生双导师制}

从2002年开始, 南通大学教育技术学专业开始实行双 导师制, 即一位学生配备两个导师, 教育技术学专业的教 
师是学生在学校四年全程的指导老师, 南通大学现代教育 技术中心的学生实践基地配备实践指导老师。教育技术学 专业的教师基本是在课堂上和实验室从事教育技术的理 论与实践，现代教育技术中心的实践指导老师一直在教育 技术服务教学，拥有丰厚的实践经验。本科生双导师制的 配备能够有效利用学校教育技术学人力资源, 发挥理论与 实践相结合的优点, 优化专业世家能力的培养。[9]

在导师的指导下, 借鉴研究生导师分配的方法, 尽量 尝试采用双向选导师制, 这样可以取得较好的效果。即可 以利用在新生的师生见面会上, 让学生了解各个专业任课 老师和实践指导老师的研究方向、学术背景、爱好、对学 生要求等, 然后等学生彻底了解自己的兴趣爱好后根据自 身的兴趣、爱好选择适合自己的导师, 最后再做适当的调 整。双向选择导师可以有利于发挥指导老师的专业特长, 同时可以促进学生的个性化发展。具体做法是：第一阶段 （大学一年级）：对新生进行思想引导和心理疏导, 生涯 规划设计指导、选择课程和专业方向; 第二阶段（大学二 年级）：指导制定专业发展目标、理清需要系统学习的专 业知识, 培养学生的自主学习能力和研究性学习的能力; 第三阶段（大学三年级）：指导学生参与社会实践和科研 创新活动, 培养实践能力和创新能力; 第四阶段 (大学四 年级)：做好学生实习、就业、考研等指导工作, 指导学 生完成较高质量的毕业论文。

\section{4. 加强学生的创新能力和实践能力}

\subsection{1. 组织兴趣小组}

根据学生的个人爱好和能力, 由学生自己自愿组成的 兴趣小组, 并且给学生提供技术指导和实验资源, 鼓舞学 生自主学习, 最终实现创新活动的共享和发展。[10]在信 息环境下, 根据个人兴趣的学习将会成为一种有效的学习 方式。在教学中, 依据学生的兴趣进行分组, 使得在师生 之间、学生之间能够沟通和学习, 并且培养小组的合作能 力, 来适应社会人才的需要, 成为栋梁之才。

\subsection{2. 学科竞赛平台建设}

为了促进学生学习的主动性, 开展学科竞赛平台是必 不可少的, 引导学生参加各种关于信息技术的竞赛活动, 培养学生的实践能力和团队合作精神。鼓舞学生参加全国 甚至国际的各种学科竞赛, 在竞赛项目中包括选题、申报、 总结等环节对学生的指点, 培养学生的实践能力, 鼓舞同 学们积极参与其中, 使其更好的发展。

\section{5. 建设完善的校内外实训基地, 构成适当的实践组织 形式}

因为教育技术学是一门建立在实践基础上的学科, 实 训对于教育技术学专业来说是必不可少的, 所以建设完善 的校内外实训基地, 构成适当上午时间组织形式, 进而提 高学生的动手实践能力。

\subsection{1. 建设完善的校内实训基地}

校内实训是根据学校提供的条件让学生一边学习一 边实践来提高学生的实践能力。校内实训包括实验、上机
和微格教学。（1）上机。因为教育技术学专业与网络和 计算机互不分开, 所以上机是校内实训的关键, 课件开发、 网页设计、C语言程序设计等等都是通过上机操作来练习, 从而可以让学生掌握知识和技能, 促进对知识的理解和运 用能力。（2）实验。实验可以分为基础实验和综合实验。 基础实验是指于数码相机、摄像机多媒体设备的操作能力 和局域网配置等。综合实验是指在理论知识加以创新和应 用, 例如摄影创作、电视专题片创作。（3）微格教学。 微格教学对于师范类学生来说是一种很好的掌握课堂教 学技能的培训方法, 微格教学是根据信息技术课程和教学 来展开的, 每位教师指导 6 名学生, 通过教案的编写、试 讲、面试等环节, 系统的培养学生的教学技能。另外, 为 了加强微格教学, 血学生在校期间贯穿微格教学, 鼓舞组 成微格教学小组, 开放教室, 促进学生在信息条件下教学 技能的提高。

\subsection{2. 建设完善的校外实训基地}

教育技术学学科知识更新速度较快, 对师资素质、实 验设备和场地等要求比较高, 很多高校很难在较短时间内 培养学生适应社会的需求, 所以提高学生的实践能力有效 措施是建设完善的校外实训基地, 这样可以让学生在学校 期间获得更多的实践经验, 更早的接触到社会工作环境中, 有效地缩短毕业生入职适应时间。那么, 如何建设完善的 校外实训基地呢? 笔者认为应从以下几方面着手。

\section{(i). 加强与中小学校合作, 拓展实践功能基地}

传统的教育实习时间安排的不合理, 时间太短, 安排太 后。然而连续性、渐进性全程教育实践教学体系应该是从学 生入校第一天起即实施, 包括学生在校的全部过程。与此同 时, 时间安排体现一定的的连续性、渐进性优点。首先以循 序渐进的一系列实践环节例如: 见习、模拟实习、教学技能 训练等来减轻教育实习给学生带来的负担, 使他们逐步适应 教师生涯, 成为一名合格的教育实习老师。但是本来是一个 高校跟基础教育学校双方受益的合作却往往因为高校单方 面不断的索取而成为中小学的负担, 实践基地逐渐变得单一 化。因此与中小学合作需要注意以下几方面: 第一, 必须落 实双赢承诺, 能够充分体现高校理论知识价值。基层学校可 以为高校提供真实的教学环境; 高校也应该聘请一些有丰富 实践经验的技术人员和基层教师来担任高校兼职教师，提供 梳理知识体系给学生, 让学生可以掌握系统的理论知识。第 二, 专业教师通过以学科知识体系为主要内容来指导学生基 于基地的实践活动设计。院校专业教师指导学生的实践活动, 并且可以诚心邀请中小学教师参与我们的课程建设, 这样可 以让他们彻底了解高校学生的知识水平和操作能力, 熟知实 践基地活动的教学指导, 给学生安排合适的实践项目, 这样 既可以完成所要求的任务、增长实践经验, 还可以增强学生 解决问题的自信心和专业的认可度, 再也不会存在一味的认 定: 学生看起来貌似样样都会, 实际上一样都不能熟练应用 就是“累赘”。

\section{(ii). 加强与企业合作，拓展实践功能基地}

利用各方面优势资源来提高学生的实践能力, 学校应 该与电视台、IT行业等多家单位合作, 并且建立稳定的专 
业实践基地, 不仅可以可以为师生创造了实践锻炼的机会, 而且可以培养学生综合实践能力和创新能力。合作双方以 相互学习、共同提高为原则在共同协商的基础上制定实践 教学实施方案。给教育技术学专业学生提供了实习的地方。 通过长期与实习实践基地合作, 可以建议良好的伙伴关系, 还可以提高实践教学质量, 培养学生的创造思维和动手能 力具有重要的意义

\section{5. 结论}

教育技术学专业是一门实践性很强的学科, 中国教育 技术学专业的学生实践能力发展欠缺, 这可以看出中国教 育技术学人才培养方面存在着问题, 到底如何克服这些问 题, 提高学生的动手实践能力, 培养出社会需要的应用型 人才, 使毕业生能够很快的适应工作环境, 是我们不断反 思和研究的问题。

\section{参考文献}

[1] 衰克定.教育技术专业的人才实践能力培养与实践体系建 设[J].现代教育技术, 2009, (11): 131-135。
[2] 南国农.中国教育技术学专业建设的发展道路[J].电化教育 研究, 2005, (9): 3-7。

[3] 陈琳,教育技术学本科专业人才培养模式创新研究[J].中国 电化教育, 2010, (10): 21-27。

[4] 王健,李玉斌,张海燕等.教育技术学专业人才实践能力培养 [J].电化教育研究, 2012, (6): 117-120。

[5] 李红美.教育技术学专业本科生实践创新能力培养的探索 [J].电化教育研究, 2011, (1): 2-5。

[6] 马俊强.基于智慧校园的中职计算机“翻转课堂”教学模式的 构建研究[D].广西师范大学. 2017 (7): 3-4。

[7] 李波,陈邦泽.如何培养教育技术学专业学生的实践创新能 力[J].西藏教育, 2011, (7): 3-7。

[8] 王志军,王雪.教育技术专业本科生实践能力培养模式探析 [J].实验技术与管理, 2014, (2): 2-4。

[9] 刘青荣.教学情绪的结构、现状及其影响效应 $[\mathrm{D}]$.上海师范 大学. 2018。

[10] 李志河,黄云芳,蔔婷等. 我国教育技术学本科专业培养目标 与学生就业断层因素分析 [J]. 现代教育技术, 2012, (8): $47-50$ 。 